\title{
Development of cell models for high-throughput screening system of Charcot-Marie-Tooth disease type 1
}

Yu-Ri Choi ${ }^{1,2,+}$, Sung-Chul Jung ${ }^{2,+}$, Jinhee Shin ${ }^{1}$, So Young Yoo ${ }^{1}$, Ji-Su Lee ${ }^{1}$, Jaesoon Joo ${ }^{1}$, Jinho Lee', Young Bin Hong ${ }^{1,3, *}$, and Byung-Ok Choi ${ }^{1,3, *}$

${ }^{1}$ Department of Neurology and ${ }^{3}$ Stem Cell \& Regenerative Medicine Center, Samsung Medical Center, Sungkyunkwan University School of Medicine, Seoul, Korea, ${ }^{2}$ Department of Biochemistry, Ewha Womans University School of Medicine, Seoul, Korea

Purpose: Charcot-Marie-Tooth disease (CMT) is a peripheral neuropathy mainly divided into CMT type 1 (CMT1) and CMT2 according to the phenotype and genotype. Although molecular pathologies for each genetic causative have not been revealed in CMT2, the correlation between cell death and accumulation of misfolded proteins in the endoplasmic reticulum (ER) of Schwann cells is well documented in CMT1. Establishment of in vitro models of ER stress-mediated Schwann cell death might be useful in developing drug-screening systems for the treatment of CMT1.

Materials and Methods: To develop high-throughput screening (HTS) systems for CMT1, we generated cell models using transient expression of mutant proteins and chemical induction.

Results: Overexpression of wild type and mutant peripheral myelin protein 22 (PMP22) induced ER stress. Similar results were obtained from mutant myelin protein zero (MPZ) proteins. Protein localization revealed that expressed mutant PMP22 and MPZ proteins accumulated in the ER of Schwann cells. Overexpression of wild type and L16P mutant PMP22 also reduced cell viability, implying protein accumulation-mediated ER stress causes cell death. To develop more stable screening systems, we mimicked the ER stress-mediated cell death in Schwann cells using ER stress inducing chemicals. Thapsigargin treatment caused cell death via ER stress in a dose dependent manner, which was measured by expression of ER stress markers.

Conclusion: We have developed genetically and chemically induced ER stress models using Schwann cells. Application of these models to HTS systems might facilitate the elucidation of molecular pathology and development of therapeutic options for CMT1.

Key words: Charcot-Marie-Tooth disease, Endoplasmic reticulum stress, Human PMP22 protein, Myelin P0 protein, Thapsigargin.

Received: 5 September 2014, Revised: 20 November 2014, Accepted: 8 December 2014, Published: 30 June 2015

${ }^{*}$ Corresponding authors: Byung-Ok Choi, M.D.

Department of Neurology, Samsung Medical Center, Sungkyunkwan University School of Medicine, 81 Irwon-ro, Gangnam-gu, Seoul 135-710, Korea.

Tel: +82-2-3410-1296, Fax: +82-3410-0052, E-mail: bochoi77@hanmail.net

Young Bin Hong, Ph.D.

Stem Cell \& Regenerative Medicine Center, Samsung Medical Center, 81 Irwon-ro, Gangnam-gu, Seoul 135-710, Korea.

Tel: +82-2-2148-9497, Fax: +82-3410-0052, E-mail: youngbinhong@gmail.com

these authors contributed equally to this study.

Conflict of interest: We declare that we do not have any conflicts of interests.

(c) This is an open-access article distributed under the terms of the Creative Commons Attribution Non-Commercial License (http://creativecommons.org/licenses/by-nc/4.0/) which permits unrestricted non-commercial use, distribution, and reproduction in any medium, provided the original work is properly cited.

(c) Copyright 2015 by the Korean Society of Medical Genetics 


\section{Introduction}

Charcot-Marie-Tooth disease (CMT) is a genetically and clinically heterogeneous disorder of the peripheral nervous system characterized by progressive extremity muscle degeneration and loss of sensory function [1-4]. CMT is divided into many types according to clinical phenotypes, genetic causes, and mode of inheritance. However, it is mainly divided into CMT type 1 (CMT1) and CMT type 2. The former is caused by genetic defects in Schwann cells and the latter is attributed to an anomaly in peripheral neurons [5].

More than 70 genes have been identified as a cause of CMT. Among them are 14 mutations known to cause a malfunction of Schwann cells [http://www.molgen.ua.ac.be/cmtmutations/]. The most prevalent CMT1 mutations occur in peripheral myelin protein 22 (PMP22) and myelin protein zero (MPZ). In addition, duplication or triplication of PMP22 by genetic recombination also causes CMT1 [6,7].

PMP22 and MPZ are myelin components of Schwann cells and their expression is restricted to Schwann cells. The PMP22 gene encodes a $22-k D$ protein comprising about $5 \%$ of myelin in the peripheral nervous system. It is expressed in the compact portion of essentially all myelinated fibers $[8,9]$. MPZ is the major structural protein of peripheral myelin and comprises 50\% of the protein in the sheath of peripheral nerves. Its function is to stabilize the myelin assembly by linking adjacent lamellae $[10,11]$.

Although the pathological mechanisms of CMT are not well defined, and might be heterogeneous based on the involved genes, the majority of CMT1 caused by PMP22 and MPZ mutations is related to mutant protein accumulation in the endoplasmic reticulum (ER) due to improper folding [12-14]. In fact, $80 \%$ of wild type PMP22 protein is retained in the ER due to its structural instability [15]. Thus mutation of both PMP22 and MPZ, and overproduction of PMP22, causes ER stress-mediated cell death of Schwann cells $[16,17]$.

In this context, understanding ER stress caused by PMP22 and MPZ proteins, and targeting the bypass of ER stress-mediated cell death, might be prerequisite for the treatment of CMT1. Here, we generated genetically and chemically induced ER stress models using Schwann cells as a basis for the development of therapeutic options for CMT1.

\section{Materials and Methods}

\section{Cell culture}

Primary Schwann cells were isolated from Sprague Dawley rats at postnatal day 4 according to Brockes et al. [18] with some modification. Isolated Schwann cells were cultured with 1\% fetal bovine serum (FBS), 1\% penicillin-streptomycin (PS), low glucose Dulbecco's modified eagle medium (DMEM; Biowest, Nuaille, France), N2 supplement (Life Technologies, Rockville, MD, USA), neuregulin, and forskolin (Sigma, St. Louis, MO, USA). The rat Schwann cell line S16 (ATCC, CRL-2941; Manassas, VA, USA) and HEK293 cells were cultured with 10\% FBS, 1\% PS, and high glucose DMEM. All experiments were conducted according to protocols approved by the Institutional Animal Care and Use Committees of Samsung Medical Center (2014-112-0006).

Table 1. List of primers for generation of wild type and mutant PMP22 and MPZ clones

\begin{tabular}{|c|c|c|}
\hline Experiment & Primer name & Sequence \\
\hline \multirow[t]{4}{*}{ cDNA cloning } & PMP22-F & 5'-CCGCCAGAATGCTCCTCCTGTTGCT-3' \\
\hline & PMP22-R & 5'-ТTТТСССТTССТСССТTССС-3' \\
\hline & MPZ-F & 5'-CTATGGCTCCTGGGGCTCCCTC-3' \\
\hline & MPZ-R & 5'-CTATTTCTTATCCTTGCGAGACT-3' \\
\hline \multirow[t]{10}{*}{ Mutagenesis } & PMP22-L16P-F & 5'-TCGTCCTCCACGTCGCGGTGCCGGTGCTGCTGTTC-3' \\
\hline & PMP22-L16P-R & 5'-GTGGAGACGAACAGCAGCACCGGCACCGCGACGTGG-3' \\
\hline & MPZ-V169fs-F & 5'-CGGGGGTGTCCTCGGGGTGGGCTGTTGCTGCTGC-3' \\
\hline & MPZ-V169fs-R & 5'-AAAAGCAGCAGCAGCAACAGCCCACCCCGAGGAC-3' \\
\hline & MPZ-L184fs-F & 5'-CGTGGTTCGGTACTGCTGGGCGCAGGCAGGCGGC-3' \\
\hline & MPZ-L184fs-R & 5'-CTGCAGGGCCGCCTGCCTGCGCCCAGCAGTACCG-3' \\
\hline & MPZ-R185fs-F & 5'-GGTTCGGTACTGCTGGCTACCAGGCAGGCGGCCC-3' \\
\hline & MPZ-R185fs-R & 5'-CTCTGCAGGGCCGCCTGCCTGGTAGCCAGCAGTA-3' \\
\hline & MPZ-S226fs-F & 5'-GTATGCAATGCTGGACCACCAAGCAGAAGCACCA-3' \\
\hline & MPZ-S226fs-R & 5'-ACAGCTTTGGTGCTTCTGCTTGGTGGTCCAGCAT-3' \\
\hline
\end{tabular}

PMP22, peripheral myelin protein 22; MPZ, myelin protein zero. 


\section{Construction of $P M P 22$ and $M P Z$ genes}

To obtain the PMP22 gene, cDNA was synthesized using Superscript reverse transcriptase (Invitrogen, Carlsbad, CA, USA) from total mRNA from HEK293 cells. Polymerase chain reaction (PCR) was then performed using the cDNA as a template. The MPZ gene was amplified from the pCMV6-entry-MPZ vector (Origene, Rockville, MD, USA). The amplified PCR product was cloned into an expression vector, pCMV-myc (Clontech, Mountain View, CA, USA). Mutant genes were generated using the QuikChange Site-Directed Mutagenesis Kit (Stratagene, La Jolla, CA, USA). All primer sequences are listed in Table 1.

\section{Transfection of cloned genes}

To express wild type and mutant PMP22 and MPZ genes, S16 and HEK293 cells were transfected with PMP22 and $M P Z$ containing vectors using Lipofectamine 3000 reagent (Invitrogen) according to the manufacturer's protocol. After overexpression of the genes for 48 hours, cells were harvested for further investigation.

\section{Measurement of cell viability}

HEK293 cells $\left(4 \times 10^{4}\right)$ seeded on 24 well plates were transfected with the indicated amounts of wild type and L16P mutant PMP22 DNA for 72 hours. Rat primary Schwann or $\mathrm{S} 16$ cells $\left(3 \times 10^{3}\right)$ seeded on 96 well plates were treated with ER stress inducers such as calcium ionophore A23187 (CI), brefeldin A (BFA), thapsigargin (TG), or tunicamycin (TM; Sigma) for 24 hours. Then cell viability was measured using the 3-(4,5-dimethylthiazol-2-yl)-2,5-diphenyltetrazolium bromide (MTT) assay. Briefly, cells treated with ER stress inducers were incubated with $10 \mathrm{mM}$ MTT solution for 2 hours then lysed with dimethyl sulfoxide. Relative numbers of viable cells were determined using absorbance at $560 \mathrm{~nm}$.

\section{Western blotting}

Expression of $P M P 22$ and $M P Z$ proteins were confirmed using standard Western blotting. HEK293 or S16 cells $\left(3 \times 10^{5}\right)$ were transfected with expression vectors then total cell lysate was harvested with RIPA lysis buffer (Thermo Scientific, Rockford, IL, USA). After quantification, proteins were separated and transferred onto polyvinylidene fluoride membranes. Anti-myc (Abcam, Cambridge, UK), anti-actin, anti-mouse secondary, and anti-rabbit secondary antibodies (Sigma), as well as ECL plus Western blotting substrate (Thermo Scientific), were used for detection of proteins. To determine ER stress induced by either overexpression of the proteins or by ER stress inducing chemicals, anti-heat shock $70 \mathrm{kDa}$ protein 5 (BiP), anti-DNA damage inducible transcript 3 (CHOP), and anti-eukaryotic translation initiation factor 2-alpha kinase 3 (PERK) antibodies (Cell Signaling Technology, Danvers, MA, USA) were used. Antipoly (ADP-ribose) polymerase 1 (PARP1) antibody (Cell Signaling Technology) was also used to determine apoptotic cell death.

\section{Immunocytochemistry}

Mutant protein cellular localization was determined by immunocytochemistry using anti-myc antibody (Abcam) after transfection of PMP22-L16P and MPZ- R185fs vectors into S16 cells. 4',6-diamidino-2-phenylindole (DAPI) and anti-protein disulfide isomerase (PDI) antibody (Cell Signaling Technology) were used for nuclear and ER detection, respectively. Then myc-tagged proteins were visualized by fluorescent secondary antibodies (Life Technologies).

\section{Results}

\section{Generation of genetically induced ER stress models}

To generate an ER stress model in Schwann cells, we cloned the most prevalent PMP22 and MPZ mutations. We selected the L16P mutation in PMP22, and V169fs, L184fs, R185fs, and S226fs mutations in MPZ. To effectively detect expression of the proteins, a myc-tag was fused to the $\mathrm{N}$-terminus of each protein. Expression of the mutant proteins, as well as wild type proteins, was determined using standard Western blotting after transfection of the vectors into HEK293 cells (Fig. 1). To determine whether expression of PMP22 mutant proteins induced ER stress, we compared PERK and BiP expression levels. Overexpression of both wild type and L16P mutant PMP22 elevated ER stress markers in a dose dependent manner (Fig. 1A). On the other hand, expression of wild type MPZ did not change the level of ER stress markers (Fig. 1B). Responses to mutant proteins also varied. V169fs, R185fs, and S226fs mutants induced the expression of ER stress markers, while L184fs mutant did not alter the level of BiP or CHOP (Fig. 1B). To further investigate the effect of wild type and mutant PMP22 mediated ER stress, cell viability was measured after transfection. MT assay results revealed cell death was induced by both forms of PMP22 in a dose dependent manner (Fig. 1C).

Immunocytochemical analysis was used to confirm whether the changes of ER stress markers are related to ER retention. Both mutant proteins, PMP22-L16P and MPZ-R185fs, colocalized with PDI, an ER resident protein, in S16 cells (Fig. 2). Therefore, these data imply that expression of PMP22 and 
A

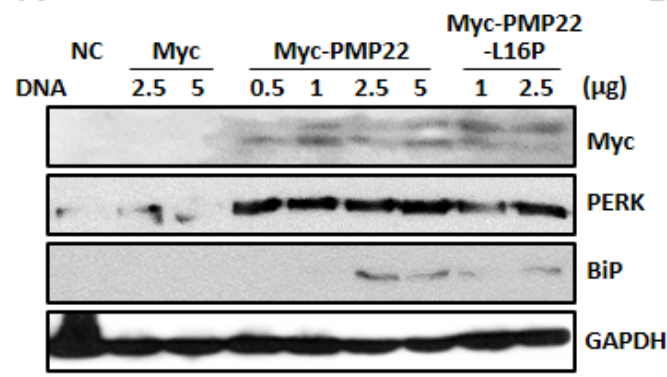

B

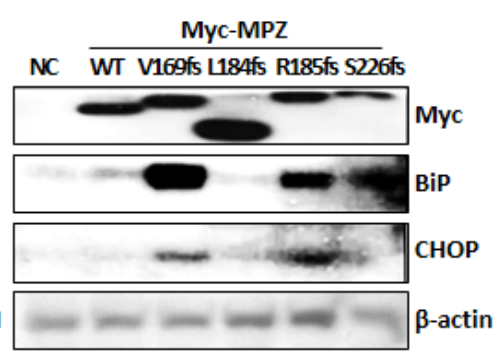

C

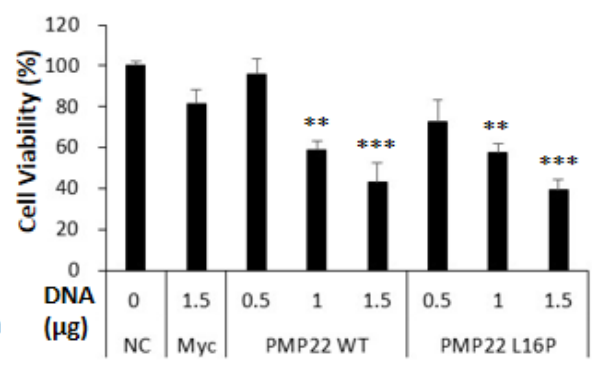

Fig. 1. Elevation of endoplasmic reticulum (ER) stress marker levels by expression of peripheral myelin protein 22 (PMP22) and myelin protein zero (MPZ) proteins. (A) ER stress markers are induced by wild type and mutant PMP22 in a dose dependent manner. The indicated amount of DNA was transfected into HEK293 cells for 48 hours. (B) Mutant MPZ protein expression induced ER stress markers in HEK293 cells. (C) Expression of wild type and L16P mutant PMP22 resulted in cell death in HEK293 cells. Cell viability was determined by the MTT assay as described in the Materials and Methods section. Three independent experiments were performed and statistical significance was compared with Myc-control DNA using the Student's t-test. Data are presented as mean \pm standard error of mean. ${ }^{\star \star} P<0.01,{ }^{\star \star \star} P<0.001$. NC, no transfection control.

A
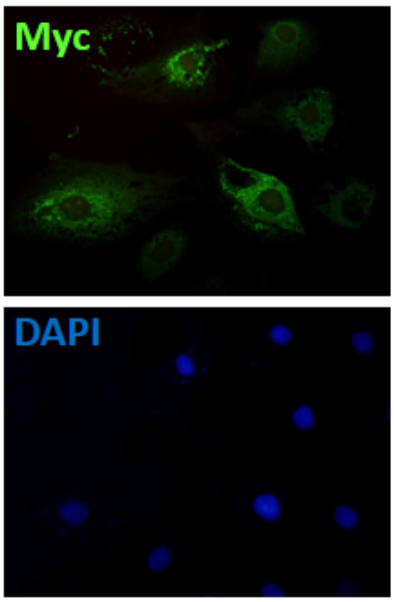

B
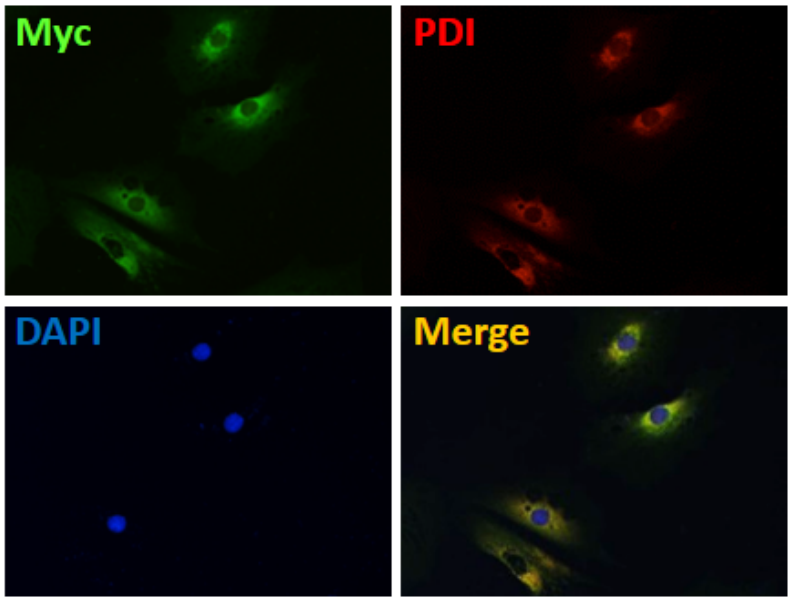

Fig. 2. Localization of mutant peripheral myelin protein 22 (PMP22) and myelin protein zero (MPZ) proteins. Myc-tagged PMP22L16P (A) and MPZ-R185fs (B) DNA was transfected into S16 cells for 24 hours. Localization of each protein was determined using immunocytochemistry. Anti-protein disulfide isomerase (PDI) antibody and 4',6-diamidino-2-phenylindole (DAPI) were used to indicate endoplasmic reticulum and nuclear localization, respectively.
MPZ mutant forms causes ER retention resulting in ER stress in Schwann cells.

\section{Generation of chemically induced ER stress models}

Since accumulation of mutant PMP22 and MPZ proteins leads to ER stress, we next sought to establish a more stable cell model for further development of high-throughput screening (HTS) by mimicking genetic models. Several chemicals are reported to induce ER stress; however, none have been demonstrated in Schwann cells.

To generate a chemically induced ER stress model in Schwann cells, we treated cells with $\mathrm{Cl}, \mathrm{BFA}, \mathrm{TM}$, or TG for 24 hours. $\mathrm{Cl}$ treatment did not induce effective ER stress-mediated cell death in either primary Schwann cells or S16 cells (data not shown). However, treatment of TG (Fig. 3A), TM (Fig. 3B), and BFA (data not shown) induced cell death in a dose dependent manner in both cell types. Overall, primary Schwann cells were more vulnerable to ER stress inducers.

To determine whether reduced cell viability is correlated with ER stress responses, we measured the changes of $\mathrm{CHOP}$, an ER stress-associated apoptotic protein, after treatment with TG. Treatment with TG for 6 hours was sufficient to induce the expression of ER stress markers in both primary Schwann cells and $\mathrm{S} 16$ cells (Fig. 3C). These data demonstrated that TG, or other chemicals, can induce ER stress similar to mutant PMP22 and MPZ proteins in Schwann cells.

\section{Discussion}

There are currently no treatment options for CMT. Recently, vitamin $\mathrm{C}$ application showed promising therapeutic efficacy in animal models [19]. However, it has not been successful in 
A

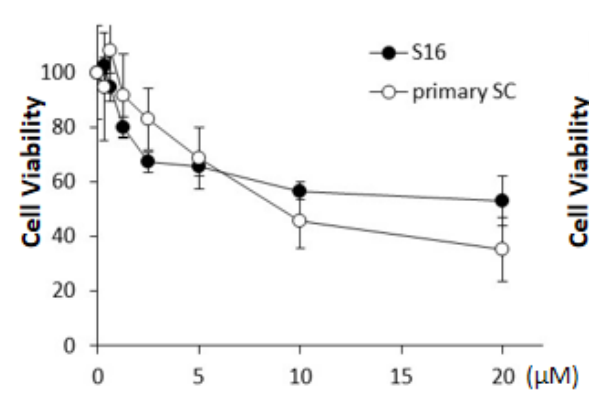

B

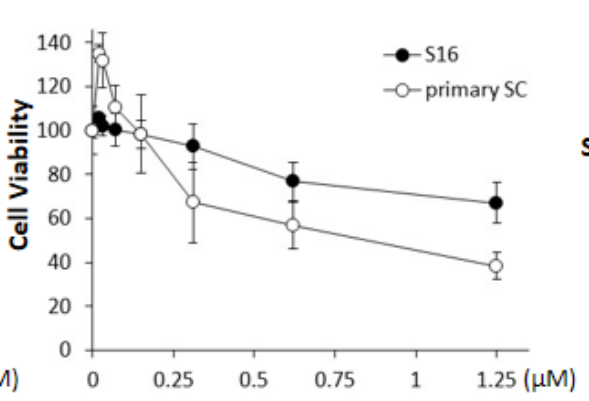

C

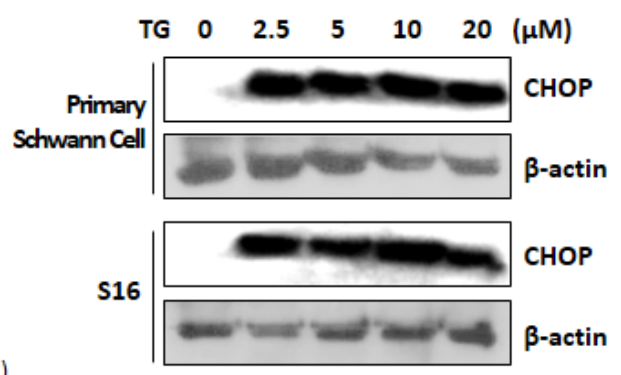

Fig. 3. ER stress-mediated cell death. The indicated concentrations of TG (A) and TM (B) were applied to primary Schwann cells and S16 cells for 24 hours. Cell viability was determined by the MTT assay. Data are representative of three independent experiments. (C) Chemically induced ER stress in both primary Schwann cells and S16 cells was determined after treatment of the indicated concentrations of TG for 24 hours. Expression levels of the ER stress marker CHOP were measured. ER, endoplasmic reticulum; TG, thapsigargin; TM, tunicamycin; SC, Schwann cell.

clinical trials [20]. Several other therapeutic strategies effective in animal models have not been clinically tried yet $[21,22]$. To assist in the development of new therapeutics, a HTS system was developed to search for PMP22 transcriptional modulators capable of addressing the gene dosage problem, which is related to duplication of the gene [23]. Using a reporter system, they screened over 3,000 chemicals and found several transcriptional modulating candidates.

Twenty PMP22 and 36 MPZ mutations have been listed in OMIM (Online Mendelian Inheritance in Man). Among them, several mutations have been well characterized to induce ER stress $[14,17]$. Structurally, ER retention mechanisms by the PMP22 L16P mutant are well explained. Induction of ER stress markers caused by MPZ mutations were also characterized. Mutant MPZ proteins are also retained in the ER and trigger unfolded protein responses (UPR) that are measured by the UPR markers $\mathrm{BiP}, \mathrm{CHOP}$, and genes downstream of $\mathrm{CHOP}$ [24].

To combat ER stress caused by PMP22 and MPZ mutations, oral administration of curcumin was tested in animal models and exhibited improvement of ER protein retention; in addition, it reduced peripheral neuropathy severity in both CMT1A and CMT1B mouse models $[17,25]$. Thus targeting ER stress might be a promising treatment option for CMT1. In this context, generation of HTS systems might be necessary for future drug development. In this study, we chose several PMP22 and MPZ mutants: L16P mutation in PMP22 and V169fs, L184fs, R185fs, S226fs mutations in MPZ. They have been well characterized in previous reports and occur in high frequencies within the genes.

In this study, we used both genetic and chemical induction systems. Transient expression of mutant proteins was enough to trigger ER stress and cell death. However, this model lacks critical options for further drug discovery. For instance, the genetically induced cells could not be used for stable cell lines to generate a reproducible system due to viability. Therefore, we explored another system to substitute the genetic model. Several chemicals, such as TG, can trigger ER stress by mimicking genetic stress [26]. Our data revealed that TG induces ER stress-mediated cell death in Schwann cells, which is the main pathological mechanism in CMT1. Therefore, chemically induced ER stress models can stably substitute mutant proteins causing ER stress.

In conclusion, we have developed a chemically induced ER stress model using Schwann cells. Application of this model to a HTS system might facilitate the development of therapeutic options for CMT1.

\section{Acknowledgements}

This study was supported by the Korean Health Technology R\&tD Project, Ministry of Health \&t Welfare (A120182) and by the National Research Foundation of Korea (NRF) grants funded by the Korean government, MSIP (NRF-2014R1A2A2A01004240) and MOE (NRF-2013R1A1A2007873).

\section{References}

1. Harding $A E$, Thomas PK. The clinical features of hereditary motor and sensory neuropathy types I and II. Brain 1980;103:259-80.

2. Skre H. Genetic and clinical aspects of Charcot-Marie-Tooth's disease. Clin Genet 1974;6:98-118.

3. Lupski JR, Garcia CA. Charcot-Marie-Tooth peripheral neuropathies and related disorders. In: Scriver $\mathrm{CR}$, ed. The metabolic and molecular bases of inherited disease. Volume 4. 8th ed. New York: McGraw Hill, 2001:5759-88.

4. Patzkó A, Shy ME. Update on Charcot-Marie-Tooth disease. Curr 
Neurol Neurosci Rep 2011;11:78-88.

5. Hanemann $\mathrm{CO}$. Hereditary demyelinating neuropathies: from gene to disease. Neurogenetics 2001;3:53-7.

6. Lupski JR, Garcia CA, Parry GJ, Patel PI. Charcot-Marie-Tooth polyneuropathy syndrome: clinical, electrophysiological, and genetic aspects. In: Appel SH, ed. Current neurology. Chicago: MosbyYearbook, 1991:1-25.

7. Liu P, Gelowani V, Zhang F, Drory VE, Ben-Shachar S, Roney E, et al. Mechanism, prevalence, and more severe neuropathy phenotype of the Charcot-Marie-Tooth type 1A triplication. Am J Hum Genet 2014; 94:462-9.

8. Snipes GJ, Suter U, Welcher AA, Shooter EM. Characterization of a novel peripheral nervous system myelin protein (PMP-22/SR13). J Cell Biol 1992;117:225-38.

9. Spreyer P, Kuhn G, Hanemann CO, Gillen C, Schaal H, Kuhn R, et al. Axon-regulated expression of a Schwann cell transcript that is homologous to a 'growth arrest-specific' gene. EMBO J 1991;10:36618.

10. Lemke G. Molecular biology of the major myelin genes. Trends Neurosci 1986;9:266-70.

11. Sutcliffe JG. The genes for myelin. Trends Genet 1987;3:73-6

12. Tobler AR, Notterpek L, Naef R, Taylor V, Suter U, Shooter EM. Transport of Trembler-J mutant peripheral myelin protein 22 is blocked in the intermediate compartment and affects the transport of the wild-type protein by direct interaction. J Neurosci 1999;19: 2027-36.

13. Myers JK, Mobley CK, Sanders CR. The peripheral neuropathy-linked Trembler and Trembler-J mutant forms of peripheral myelin protein 22 are folding-destabilized. Biochemistry 2008;47:10620-9.

14. Sakakura M, Hadziselimovic A, Wang Z, Schey KL, Sanders CR. Structural basis for the Trembler-J phenotype of Charcot-MarieTooth disease. Structure 2011;19:1160-9.

15. Pareek S, Notterpek L, Snipes GJ, Naef R, Sossin W, Laliberté J, et al. Neurons promote the translocation of peripheral myelin protein 22 into myelin. J Neurosci 1997;17:7754-62.

16. Sancho S, Young P, Suter U. Regulation of Schwann cell proliferation and apoptosis in PMP22-deficient mice and mouse models of
Charcot-Marie-Tooth disease type 1A. Brain 2001;124:2177-87.

17. Khajavi M, Inoue K, Wiszniewski W, Ohyama T, Snipes GJ, Lupski JR. Curcumin treatment abrogates endoplasmic reticulum retention and aggregation-induced apoptosis associated with neuropathy-causing myelin protein zero-truncating mutants. Am J Hum Genet 2005;77: 841-50.

18. Brockes JP, Fields KL, Raff MC. Studies on cultured rat Schwann cells. I. Establishment of purified populations from cultures of periphera nerve. Brain Res 1979;165:105-18.

19. Passage $E$, Norreel JC, Noack-Fraissignes $P$, Sanguedolce $V$, Pizant J, Thirion $X_{1}$ et al. Ascorbic acid treatment corrects the phenotype of a mouse model of Charcot-Marie-Tooth disease. Nat Med 2004;10:396401.

20. Pareyson D, Reilly MM, Schenone A, Fabrizi GM, Cavallaro T, Santoro

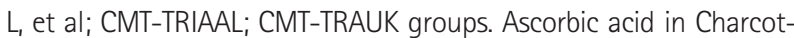
Marie-Tooth disease type 1A (CMT-TRIAAL and CMT-TRAUK): a double-blind randomised trial. Lancet Neurol 2011;10:320-8.

21. Pleasure DE, Chance PF. Neurotrophin-3 therapy for Charcot-MarieTooth disease type 1A. Neurology 2005;65:662-3.

22. Sahenk Z, Galloway G, Edwards C, Malik V, Kaspar BK, Eagle A, et al. TrkB and TrkC agonist antibodies improve function, electrophysiologic and pathologic features in Trembler J mice. Exp Neurol 2010;224:495506.

23. Jang SW, Lopez-Anido C, MacArthur R, Svaren J, Inglese J. Identification of drug modulators targeting gene-dosage disease CMT1A. ACS Chem Biol 2012;7:1205-13.

24. Pennuto $M$, Tinelli $E$, Malaguti $M$, Del Carro $U$, D'Antonio $M$, Ron $D$, et al. Ablation of the UPR-mediator CHOP restores motor function and reduces demyelination in Charcot-Marie-Tooth 1B mice. Neuron 2008:57:393-405

25. Khajavi M, Shiga K, Wiszniewski W, He F, Shaw CA, Yan J, et al. Oral curcumin mitigates the clinical and neuropathologic phenotype of the Trembler-J mouse: a potential therapy for inherited neuropathy. Am J Hum Genet 2007;81:438-53.

26. Pahl HL. Signal transduction from the endoplasmic reticulum to the cell nucleus. Physiol Rev 1999;79:683-701. 